\title{
Double-bundle Anatomic Anterior Cruciate Ligament Reconstruction Using Hamstring-bone Graft: a Long-term Follow Up
}

\section{Jingyue Gan}

Zhujiang Hospital of Southern Medical University; The Affiliated Dalian Friendship Hospital of Dalian Medical University https://orcid.org/0000-0002-5673-4746

Jie Li

Zhujiang Hospital of Southern Medical University

\section{Vidmi Taolam Martin}

Zhujiang Hospital of Southern Medical University

Ke Liu

The Affiliated Dalian Friendship Hospital of Dalian Medical University of Dalian Medical University: Dalian Municipal Friendship Hospital

Bo Yu ( $\square$ gzyubo@163.com)

Zhujiang Hospital of Southern Medical University

\section{Technical note}

Keywords: Anterior cruciate ligament injury, Hamstring tendon, Double- bundle reconstruction, Arthroscopy

Posted Date: February 23rd, 2021

DOl: https://doi.org/10.21203/rs.3.rs-231326/v1

License: (c) (1) This work is licensed under a Creative Commons Attribution 4.0 International License. Read Full License 


\section{Double-bundle anatomic anterior cruciate ligament}

\section{reconstruction using hamstring-bone graft: a long-term follow up}

Jingyue Gan ${ }^{1,2}$, Jie Li ${ }^{1}$, Vidmi Taolam Martin ${ }^{1}, \mathrm{Ke} \mathrm{liu}^{2}$, Bo Yu${ }^{1}$ *

[Abstract] Purpose We modified the hamstring tendon graft into the hamstring-bone composite graft in double-bundle anterior cruciate ligament reconstruction for anterior cruciate ligament ( $A C L)$ injuries. Here, we evaluated the technique's effectiveness by investigating the clinical results in restoring the stability and function of the knee joint. Methods We reviewed 42 patients who underwent anterior cruciate ligament reconstruction (ACLR) with the hamstring-bone graft for ACL injuries from January 2013 to April 2015 , with an average follow-up of 66.6 months. The objective evaluations (KT-1000, Lachman test and pivot-shift test) and the subjective evaluations (International Knee Documentation Committee (IKDC) scores, Lysholm scores, and Tegner scores) were performed preoperatively and postoperatively. Results Forty-one of 42 patients were negative in the Lachman test and forty of 42 patients were negative in the pivot-shift test after the operation at the final follow-up time. The IKDC, Lysholm, and Tegner scores improved significantly from $56.24 \pm 9.28$ to $85.33 \pm 3.37$, from $62.00 \pm 11.09$ to $90.43 \pm 3.71$, and from $2.3 \pm 0.87$ to $6.5 \pm 0.67$ at the final follow-up. Conclusion Double-bundle anatomic ACLR with a hamstringbone composite graft can restore the anteroposterior and rotational stability for ACL injuries. The modified technique can provide excellent clinical outcomes with a long-term follow-up.

Keywords: Anterior cruciate ligament injury; Hamstring tendon; Double- 
bundle reconstruction; Arthroscopy

\section{Introduction}

Single-bundle and double-bundle anatomic anterior cruciate ligament reconstruction (ACLR) are common techniques for $A C L$ injuries. In recent the decade, single-bundle anatomic ACLR has been widely recognized because of its similar clinical effect and easier surgical techniques than double-bundle anatomic ACLR[1-4]. However, it is still controversial whether double-bundle or single-bundle reconstruction will provide superior clinical results. Previous studies revealed no significant difference in objective or subjective outcomes between the double-bundle and single-bundle reconstruction[5-9]. Conversely, several biomechanical and clinical studies have demonstrated that double-bundle reconstruction was superior to single-bundle reconstruction in restoring the knee joint's anterior and rotational stability[10-14]. Moreover, compared with rates reported after single-bundle ACLR, double-bundle ACLR utilizing a hamstring autograft have lower graft rupture rates in patients[15-18]. Given this, we used doublebundle anatomic ACLR for ACL injuries.

Besides, ACLR with hamstring tendon requires osteointegration of the tendon graft after initial fixation. Appropriate graft-to-bone healing is essential for postoperative rehabilitation and resumption of athletic activities[19]. Some researchers have demonstrated that bone-patellar tendon-bone autografts heal more rapidly because of their bone-to-bone interfaces when the hamstring autograft's soft tissue-to-bone interface takes $9 \sim 12$ weeks to heal[20-21]. Consequently, we proposed that the hamstringbone composite graft modified the hamstring tendon graft to insert the tibial tunnel, augmenting graft-bone interface healing.

This study aimed to investigate the clinical outcomes and knee joint 
stability of the patients who underwent the modified double-bundle anatomic ACLR technique with a hamstring-bone composite graft. We hypothesized that the modified technique could restore the anteroposterior and rotational stability for $A C L$ injuries. With this new technique, the patients gained excellent long-term stability and function of the knee joint.

\section{Materials and Methods}

\section{Patients}

We retrospectively reviewed 42 knees from 42 patients who underwent ACLR with hamstring-bone autograft for ACL injuries from January 2013 to April 2015. The inclusion criteria: primary ACL injuries; unilateral ACL injuries; less than 6 months after injury; no previous surgery on the affected knee; with or without concomitant meniscal tear; patients aged 18 to 45 years. The exclusion criteria include: injuries combined with a posterior cruciate ligament injury; collateral ligament and/or posterolateral complex injury; lower extremity fracture; radiographic evidence of Kellgren-Lawrence grade 3 or 4 osteoarthritis and/or severe osteoporosis; skeletal immatureness; partial ACL injuries. The study was approved by the Ethics Committee of zhujiang Hospital of Southern Medical University. An experienced senior surgeon performed all patients who underwent ACLR surgery with the hamstring-bone autograft. The knee joint stability was evaluated by means of KT-1000, Lachman test and pivot-shift test preoperatively and postoperatively. International Knee Documentation Committee score (IKDC), Lysholm scores and Tegner scores were estimated preoperatively and postoperatively. All 42 patients were followed up, with an average follow-up of $66.6 \pm 8.4$ months (ranged from 53 to 80 months). The demographic data are displayed in Table 1.

\section{Surgical technique}

Every patient was treated with combined spinal-epidural anesthesia or general anesthesia. Arthroscopy was used through the anterolateral and anteromedial portal to verify $\mathrm{ACL}$ rapture. The synovial tissue of the 
intercondylar fossa was cleaned to avoid the crowding of the intercondylar fossa. For patients with stenosis of the intercondylar fossa, the enlargement formation of the intercondylar fossa was required. After confirmation of $A C L$ rupture, a longitudinal incision of about $3 \sim 4 \mathrm{~cm}$ was made at the medial side to the tibia tuberosity. The sartorius muscle was split up along the direction of muscle fibers and pulled upward to make the semitendinosus and gracilis tendon visible. The cuboid bone block $(20 \times 10 \times 10 \mathrm{~mm}$ in size $)$ that connected semitendinosus and gracilis tendon was harvested firstly by osteotome at the site of hamstring tendon insertion(Figure 1A,1B). Then, the semitendinosus and gracilis tendons were harvested by the tendon harvester, and the bone block was divided into two equal parts $(10 \times 10 \times 10 \mathrm{~mm}$ in size)(Figure 2A,2B). Semitendinosus and gracilis tendon were performed by double loop suture technique with the NO 2 Ethibond(Ethicon) nonabsorbable sutures at both ends of the tendon and folded to create 4 strands respectively. The diameter of tendon grafts was measured precisely, and grafts were pre-tensioned under 20 pounds for 10 minutes to be ready for passing through bone tunnels. Anatomical double-tunnel double-bundle ACLR was used in operation. Semitendinosus tendon was used for reconstruction of an anteromedial bundle $(A M B)$ and gracilis tendon was used for the posterolateral bundle(PLB)(Figure 3A,3B).

The tibial PLB tunnel was drilled $5 \sim 6 \mathrm{~mm}$ away from the anterior margin of PCL, and the direction was $45^{\circ}$ in the horizontal plane and $45^{\circ}$ in the sagittal plane, and the tibial AMB tunnel was drilled $5 \sim 6 \mathrm{~mm}$ behind the anterior margin of $\mathrm{ACL}$ and the direction was $45^{\circ}$ in the horizontal plane and $20^{\circ} \sim 30^{\circ}$ in sagittal plane. Through the anteromedial portal, the $3 \mathrm{~mm}$ eccentric femoral tunnel guider was used at the posterior edge of the lateral femoral condyle, and the center of the femoral attachment of the AMB with 
the knee flexed extremely, using inside-out technique. PLB tunnel was located $5 \mathrm{~mm}$ above the lateral femoral condylar cartilage at $90^{\circ}$ of knee flexion, using the inside-out technique. The bone wall between the femoral tunnel apertures was at least 1 to $2 \mathrm{~mm}$.

After completing 2 tibial and 2 femoral tunnels in which the diameter was determined according to the measured diameter of prepared grafts, we trimmed 2 cube tendon-bone blocks into cylindrical plugs. The diameter of cylindrical bone plugs were $1 \sim 2 \mathrm{~mm}$ smaller than that of the tibial tunnel, so that the sutures at the end of the graft tendon could be pulled in the tibial tunnel frictionlessly. The PLB graft and the AMB graft were introduced through the tibial tunnel to the femoral tunnel. Finally, the PLB and AMB were fixed at $10^{\circ} \sim 20^{\circ}$ of knee flexion with mini-disc suspension, respectively(B.Braun company, Germany)(Figure 4,5).

\section{Postoperative rehabilitation}

We implemented a nonradical rehabilitation program after the operation. The range of flexion and extension of the knee joint were limited to $0 \sim 30^{\circ}$ within 2 weeks and $0 \sim 90^{\circ}$ within 4 weeks after the operation and strictly limited the activity time to 15 minutes twice per day. Isometric musclestrengthening exercises were allowed the day after surgery. Partial-body weight-bearing was started on the third week, and full weight-bearing was started on the fifth week after the operation. Running, jump-landing training and open kinetic chain exercises were allowed after 4 months, and return to sports activities was allowed after 8 months.

\section{Clinical effectiveness follow-up}

All patients were examined preoperatively and postoperatively. At the final follow-up, the knee functions were evaluated by the following items. Objective evaluations: (1)KT-1000 (MEDmetric,USA); (2)Lachman test;(3) 
Pivot-shift test. Subjective evaluations:(1)International Knee Documentation Committee(IKDC) scores[22]; (2)Lysholm scores[23]; (3)Tegner scores[24].

\section{Statistical analysis}

All continuous variables were expressed as the means \pm standard deviation. The independent samples t-test was used to compare the continuous variables between preoperation and postoperation. Numeration data were compared with the Chi-square test. SPSS statistical software 23.0 (SPSS Inc USA) was used for statistical calculations, and significance was defined as $\mathrm{P}<0.05$.

\section{Results}

We retrospectively reviewed 42 knees from 42 patients aged 18 to 45 years who underwent ACLR with the hamstring-bone autograft for $A C L$ injuries from January 2013 to April 2015. All patients were followed up. Only one patient suffered from superficial infections, and one patient suffered from incision fat liquefaction at the anteromedial tibial incision site. No deep infections, skin necrosis, or ACL re-ruptures was observed and no nonunion of the tibial tunnel was found in our study after long-term follow-up.

In respect of subjective evaluations(Table 2), the IKDC, Lysholm and Tegner scores improved significantly from $56.24 \pm 9.28$ to $85.33 \pm 3.37$, from $62.00 \pm 11.09$ to $90.43 \pm 3.71$, and from $2.3 \pm 0.87$ to $6.5 \pm 0.67$ at the last follow-up $(P<0.01)$. In respect of knee stability evaluations(Table 3 ), the mean value of KT-1000(MED metric, USA, values of $134 \mathrm{~N}$ under preload at $30^{\circ}$ of knee flexion) decreased from $7.0 \pm 0.90$ preoperatively to $1.1 \pm 0.41$ postoperatively $(\mathrm{P}<0.01)$. The postoperative Lachman test results showed that $97.6 \%$ (41 cases) patients performed negative results when $2.4 \%$ (1 case) patients performed positive results. The postoperative pivot-shift test results showed that $95.2 \%$ (40 cases) patients performed negative results 
when $4.8 \%$ ( 2 cases) patients performed positive results.

\section{Discussion}

After a long-term follow-up, we found that the double-bundle ACLR with hamstring-bone graft not only achieved good clinical outcomes, but also restored knee joint stability. This is similar to the results of many other researches. Jarvela et al.[25] compared tunnel enlargement in sixty patients with double-tunnel and single-tunnel ACLR, and then they found that on the tibial side, tunnel enlargement was greater in the single-tunnel group than in the double-tunnel group, which demonstrated that the patients who had more tunnel enlargement had significantly more anterior and rotational laxity of the operated knee as well. Aga $C$ et al. [26] detected tunnel widening in double-tunnel and single-tunnel ACLR through a semiautomated 3D CT imaging modality, and they found a larger amount of widening in the single femoral and tibial tunnels compared to the double femoral and tibial tunnels. In our study, the Lachman and pivot-shift test showed a very low positive rate postoperatively, and we did not find $A C L$ re-rupture cases at the final follow-up. The results demonstrated that our modified technique could restore both anteroposterior stability and rotational stability. A research showed that $A C L$ re-rupture rate after double-tunnel reconstruction was significantly lower than that of single-tunnel reconstruction in patients younger than 30 years old[15]. Sasaki $S$ et al.[27]compared the efficacy of double-tunnel double-bundle and single-tunnel single-bundle ACLR and found no differences in subjective and objective indicators between the two groups. However, in the study, a rectangular-tunnel technique was used instead of a single round-tunnel for the femoral side. The author indicated that rectangular-tunnel reconstruction could provide a larger coverage at the ACL attachment area compared with single round-tunnel and result in closer to normal knee biomechanics. Therefore, the study indicates that the researcher doubts the capacity for restoring single round-tunnel reconstruction's knee stability. Based on the above research and our study, 
we consider that double-tunnel double-bundle reconstruction is superior to single-tunnel single-bundle reconstruction in restoring knee joint stability. For these reasons, if the patients are young or middle aged and high demand for physical activities, double-tunnel double-bundle ACLR will be considered necessary.

In our study, we used suspensory fixation of the hamstring tendon graft: endobutton at the exit of the femoral tunnel and mini-disc at the exit of the tibial tunnel. Fixation methods commonly used in ACLR could be included in two types[28]: (1) fixation at a distance such as TransFix and EndoButton; (2) fixation with an interference screw. At present, interface screw fixation is widely used. Weiler et al.[21]demonstrated that fixation at the joint surface using interference fixation might neutralize tendon graft-tunnel motions, thus promoting a direct type of ligament insertion at the joint surface. Weiler et al.[21] also found that the head of the interference screw which was usually inserted into the tibial tunnel in an inside-out direction could flush with the intra-articular aperture of the tibial tunnel. Currently, the interference screw is usually inserted into the tibial tunnel in an outside-in direction, and this technique can reduce the compression of the interference screw on the graft. However, graft tunnel motion at the intra-articular aperture of the tibial tunnel would be expected in this technique, especially when the length of the interference screw is shorter than the length of the tibial tunnel[21]. The suspensory fixation of hamstring tendon far from the tunnel aperture did not compress the tendon graft along the tunnel's length as fixation with an interference screw. In addition, this technique may also allow the ingrowth of blood vessels along the entire length of the tendon graft[29]. The study[30], explored the tendon-to-bone healing differences between interference screw versus EndoButton, indicating that fixation with interference screw led to an indirect ligamentous insertion and fixation with EndoButton led to viability of the graft tendon healing in the bone tunnel. Although fixation with 
EndoButton may lead to a slow healing process, the success rate of $A C L$ reconstruction with EndoButton is up to $95.8 \%[31]$.

There is no doubt that the graft's early intra-tunnel motion is considered to preclude graft tendon healing to bone, and the suspensory type of fixation could result in graft tunnel motion and may lead to slow healing process. To remedy the deficiency of suspensory fixation, we inserted the hamstringbone composite graft into the tibial tunnel. It has the following advantages: Firstly, the trimmed cylindrical bone plug can inosculate with the tibial tunnel's bone wall, and the healing type of the tibial tunnel graft changes from tendon-bone healing to the combination of tendon-bone healing and bone-to-bone healing. The success of ligament reconstruction procedures using tendon grafts is based on the graft incorporation between the tendon and bone. It has been reported that the bone-bone interface healed more rapidly than the soft-to-bone interface of the hamstring-tendon autograft[20, 21]. Therefore, the trimmed cylindrical bone plug promotes incorporation between graft and bone in the tibial tunnel. Secondly, the trimmed cylindrical bone plug can play a filling and occupying a role in neutralizing tendon grafttunnel motions. Some researchers suggest progressively greater grafttunnel motion in suspensory fixation as the distance from the fixation point increases, resulting in greater graft-tunnel motion at the tunnel aperture than at the tunnel exit for a graft fixed outside of the tunnel[32-34]. Suspensory fixation methods have been said to exhibit longitudinal motion due to the distance between the graft and the point of fixation, and they will lead to bone tunnel enlargement and influence knee stability[35]. In consideration of our research, the cylindrical bone plug located at the tibial tunnel can play a filling and occupying role. The distal section of the tibial tunnel, where the sutures were originally occupied, was filled with cylindrical bone plug. As a result, the cylindrical bone plug could enhance the consistent graft tension 
through squeezing the graft sutures between the end of the graft and the mini-disc at the tibial tunnel exit. Consequently, the cylindrical bone plug augments the capacity of graft fixation as the supplementary of mini-disc at tibial tunnel exit. The filling and fixation effect of cylindrical bone plug decreased the magnitude of graft tunnel motion and increased the graft's stability in the tibial tunnel. It is noted that the diameter of the graft sutures between the end of graft and the button were approximately $1 \sim 2 \mathrm{~mm}$. In order to make the graft sutures go through the gap between cylindrical bone plugs and tunnel wall more smoothly, the diameter of cylindrical bone plugs should be trimmed $1 \sim 2 \mathrm{~mm}$ smaller than that of the tibial tunnel.

In our study, there were 1 case of superficial infection and 1 case of incision fat liquefaction. The superficial infection occurred one week after the operation, and healed after limited debridement and dressing change. One patient suffered from incision fat liquefaction on the 10th day after the operation, and the wound healed after radical debridement and dressing change. In our study, we had a very low incidence of perioperative complications, so the bone harvesting procedure did not add additional injuries to the patients, and the bone defect would be healed $4 \sim 6$ months after operation(Figure 6A,6B).

There were some limitations in our study. Firstly, the modified technique lacked of biomechanical testing in cadaver specimens. Secondly, the precise evaluation of tendon-bone graft motion was not investigated in the present study. Thirdly, there was no case-control in our study. That would be better if we added controlled cases that undertook double-bundle ACLR with hamstring graft only. Fourthly, we did not evaluate the development of knee OA after the operation in our study. Although the patients obtained good clinical outcomes with a long-term follow up, further study is needed to 
evaluate the relationship between ACLR and knee OA development.

\section{Conclusion}

The hamstring-bone plug filled in the tibial tunnel reduced distance between the graft and the point of fixation. Consequently, our modified technique can decrease the graft longitudinal motion and reduce the tunnel enlargement. This technique also allows the ingrowth of blood vessels along the tendon graft's entire length compared with the interference screw fixation. After long-term follow-up, we have confirmed that double-bundle anatomic ACLR with a hamstring-bone composite graft can obtain satisfactory clinical outcomes and restore knee anteroposterior and rotational stability.

*Corresponding author: Bo Yu, MD, PhD, Assistant Professor, Department of Orthopedics, Zhujiang Hospital of Southern Medical University, Guangzhou 510282, Guangdong Province, China.gzyubo@163.com

1. Department of Orthopedics, Zhujiang Hospital of Southern Medical University, Guangzhou 510282, Guangdong Province, China

2. Department of orthopedics, The Affiliated Dalian Friendship Hospital of Dalian Medical University, Dalian 116000, Liaoning Province, China 
Table 1 Patient demographic data

\begin{tabular}{|c|c|c|c|c|c|c|c|}
\hline Gender & Ages(years) & Injury knee & Weight(Kg) & Height(cm) & $\begin{array}{c}\text { Time from injury } \\
\text { to }\end{array}$ & $\begin{array}{l}\text { Meniscus } \\
\text { injury }\end{array}$ & Follow-up(months) \\
\hline Male/female & & Right/left & & & surgery(months) & $\mathrm{M} / \mathrm{L} / \mathrm{Bi} / \mathrm{N}$ & \\
\hline $33 / 9$ & $32.9 \pm 7.1$ & $24 / 18$ & $72.1 \pm 8.4$ & $171.9 \pm 6.5$ & $12.0 \pm 6.4$ & $10 / 7 / 5 / 20$ & $66.6 \pm 8.4(53-80)$ \\
\hline
\end{tabular}

M: medial meniscus injury, L: lateral meniscus injury, Bi: bilateral meniscus injury,

$\mathrm{N}$ : none meniscus injury

Table 2 subjective evaluations of patients

\begin{tabular}{llll}
\hline & IKDC scores & Lysholm scores & Tegner scores \\
\hline Preoperative & $56.24 \pm 9.28$ & $62.00 \pm 11.09$ & $2.3 \pm 0.87$ \\
Postoperative & $85.33 \pm 3.37$ & $90.43 \pm 3.71$ & $6.5 \pm 0.67$ \\
P value & 0.000 & 0.000 & 0.000 \\
\hline
\end{tabular}

Postoperative follow-up compared with preoperative, $P<0.01$.

Table 3 objective evaluations of patients

\begin{tabular}{|c|c|c|c|c|c|}
\hline & \multirow[t]{2}{*}{$\begin{array}{l}\text { KT-1000(mm) } \\
\text { (30 of knee flexion) }\end{array}$} & \multicolumn{2}{|c|}{ Lachman testing(cases) } & \multicolumn{2}{|c|}{$\begin{array}{l}\text { Pivot-shift } \\
\text { testing(cases) }\end{array}$} \\
\hline & & Negative & Positive & Negative & Positive \\
\hline preoperative & $7.0 \pm 0.90$ & 0 & 42 & 13 & 29 \\
\hline postoperative & $1.1 \pm 0.41$ & 41 & 1 & 40 & 2 \\
\hline P value & 0.000 & 0.000 & & 0.000 & \\
\hline
\end{tabular}

Postoperative follow-up compared with preoperative, $\mathrm{P}<0.01$. 


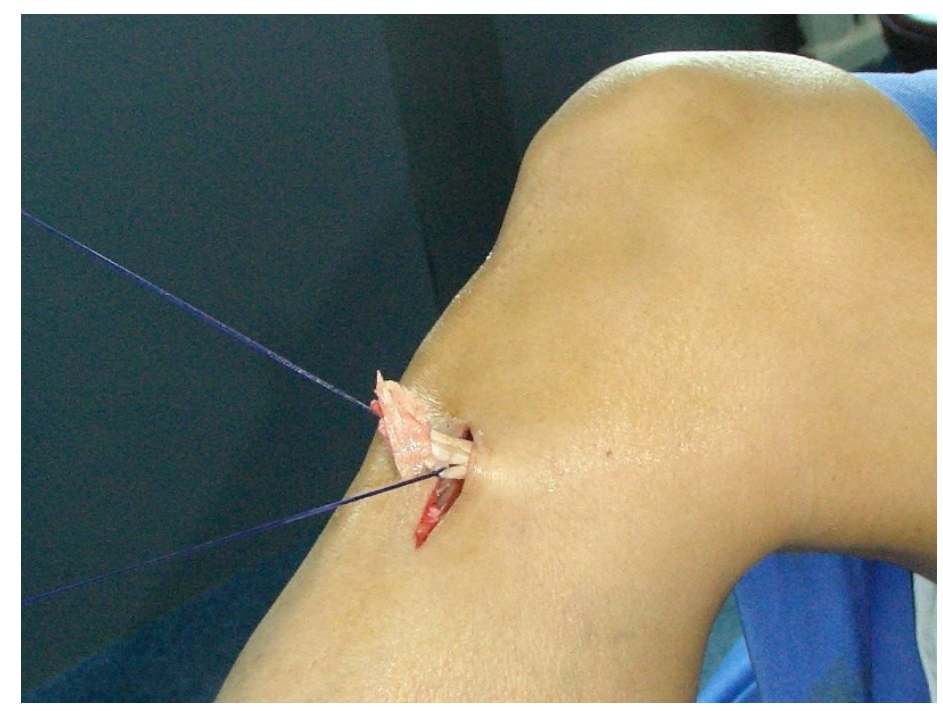

Figure 1A. Harvest of cuboid bone block at the footprint of GT and ST. GT: gracilis tendon; ST: semitendinosus tendon.

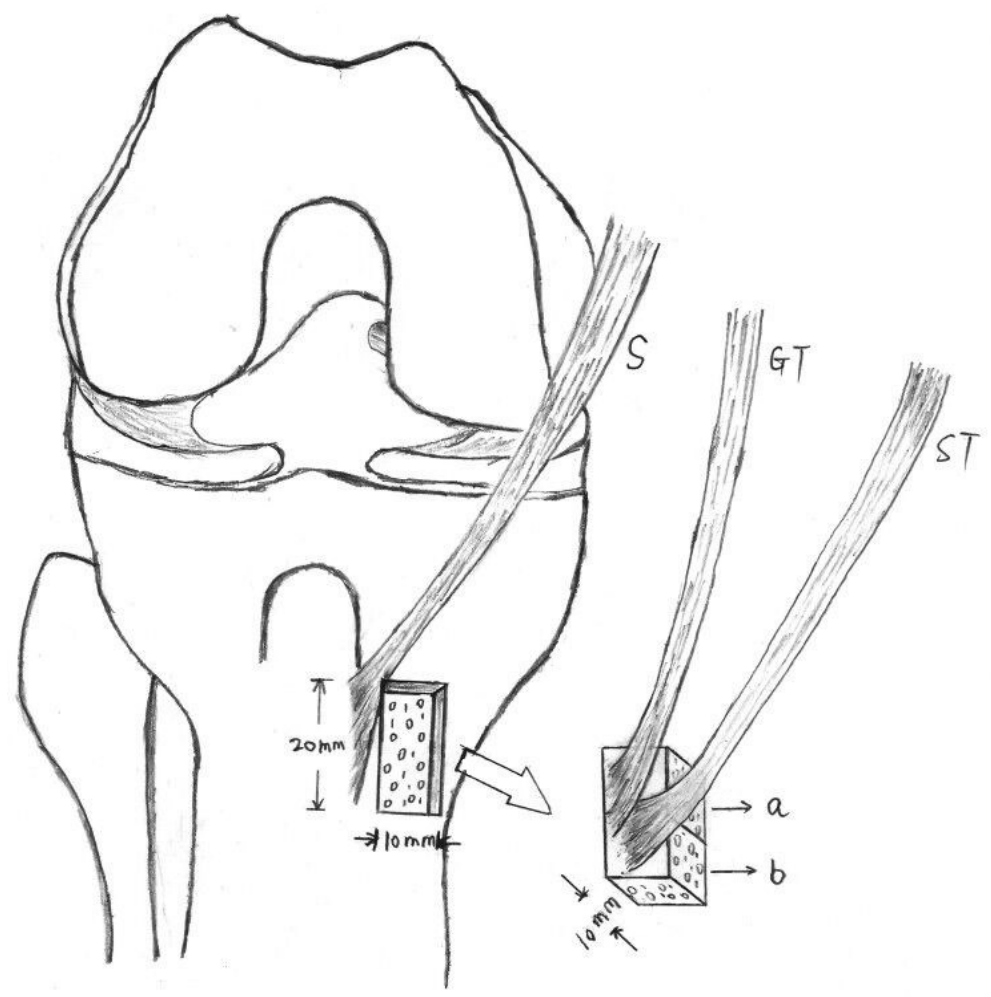

Figure $1 \mathrm{~B}$. The block is $20 \mathrm{~mm}$ length, $10 \mathrm{~mm}$ width, and $10 \mathrm{~mm}$ thickness, and it is cut into 2 equal pieces (a, b). 


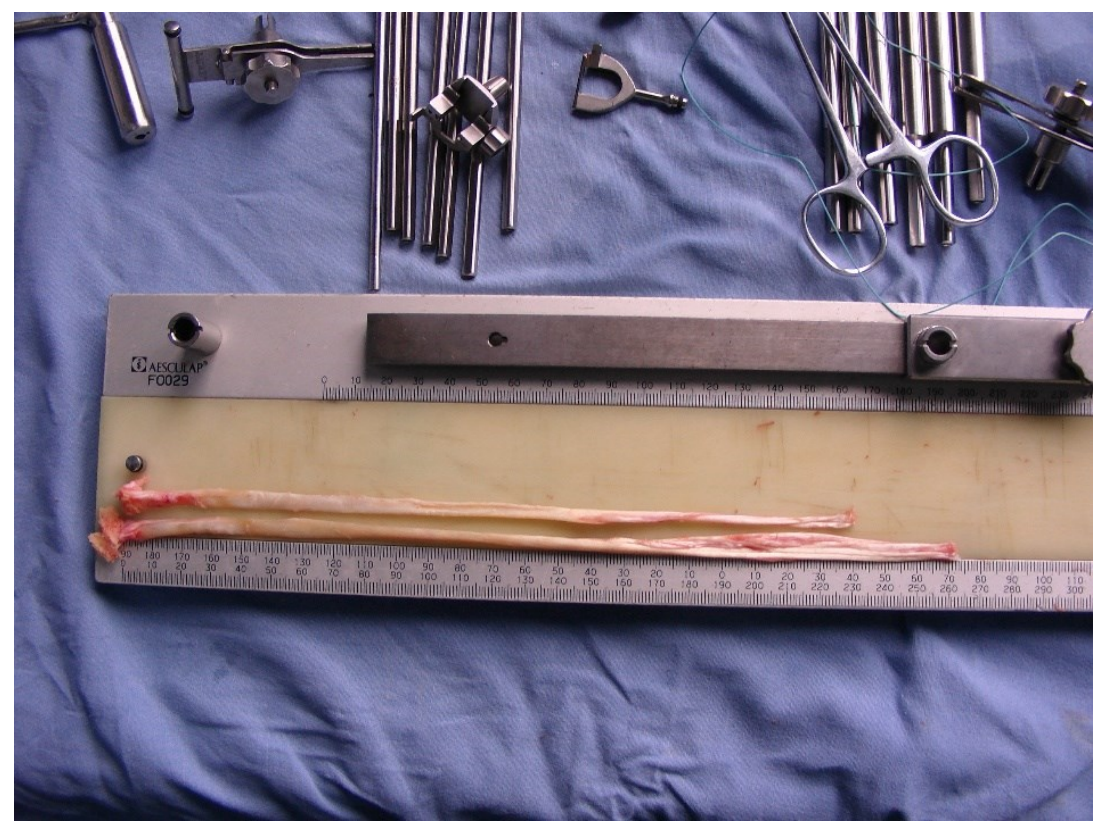

Figure 2A. Harvest of ST and GT, and the bone block is divided into two equal parts.
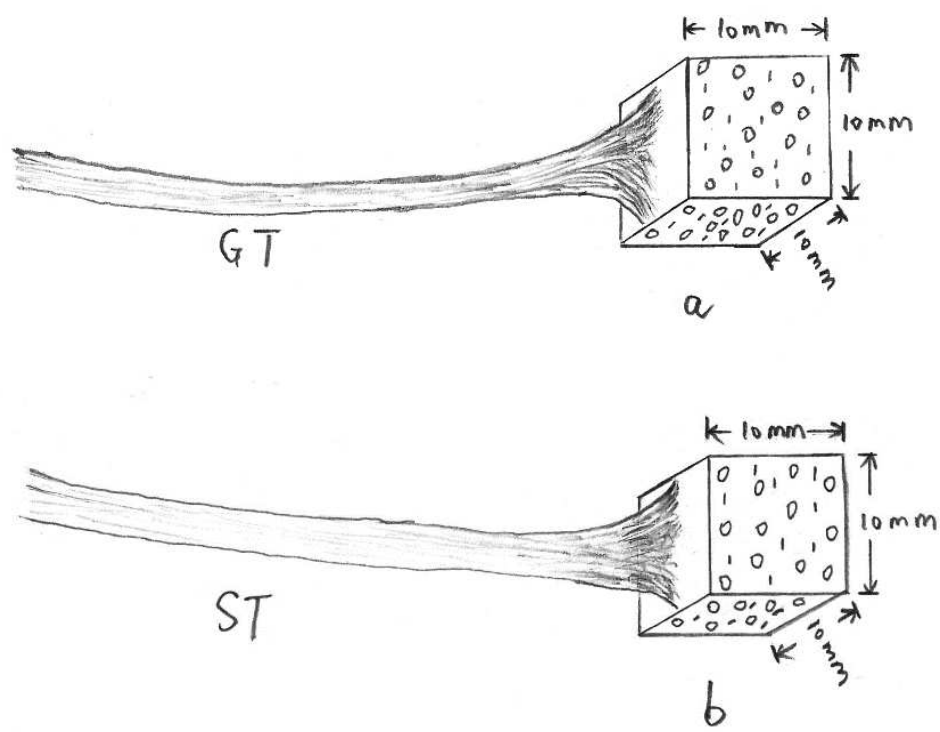

Figure 2B. The bone block is divided into two equal parts. The blocks are $10 \mathrm{~mm}$ length, $10 \mathrm{~mm}$ width, and $10 \mathrm{~mm}$ thickness, respectively. 


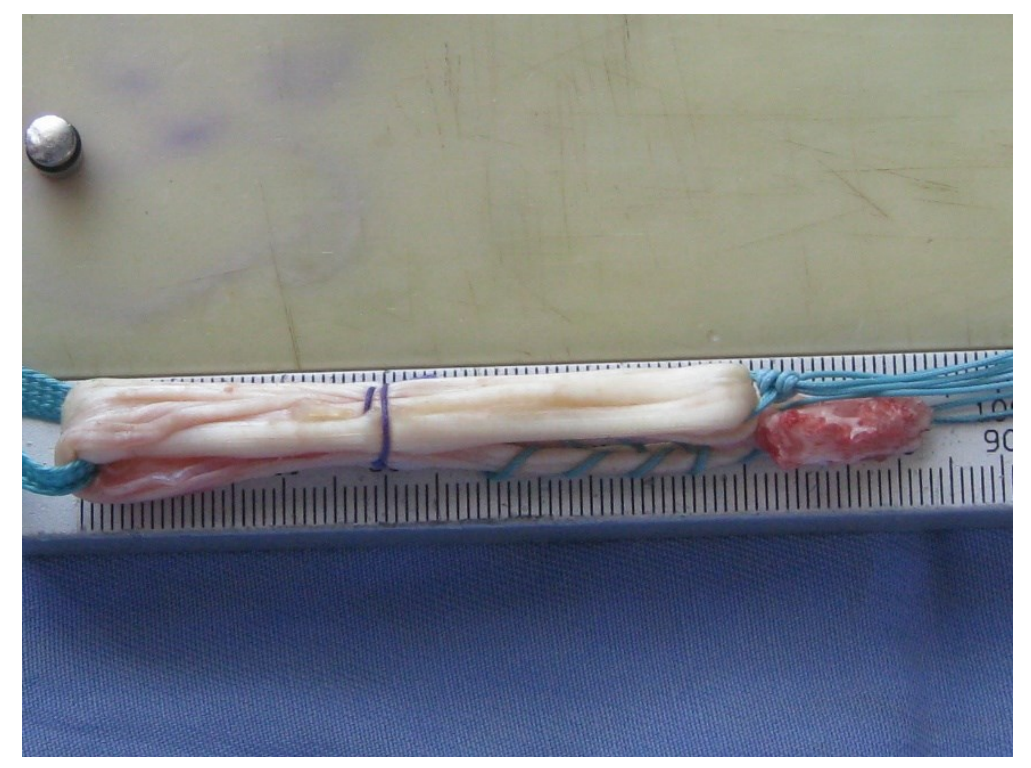

Figure 3A. Suture and fold of GT and ST.
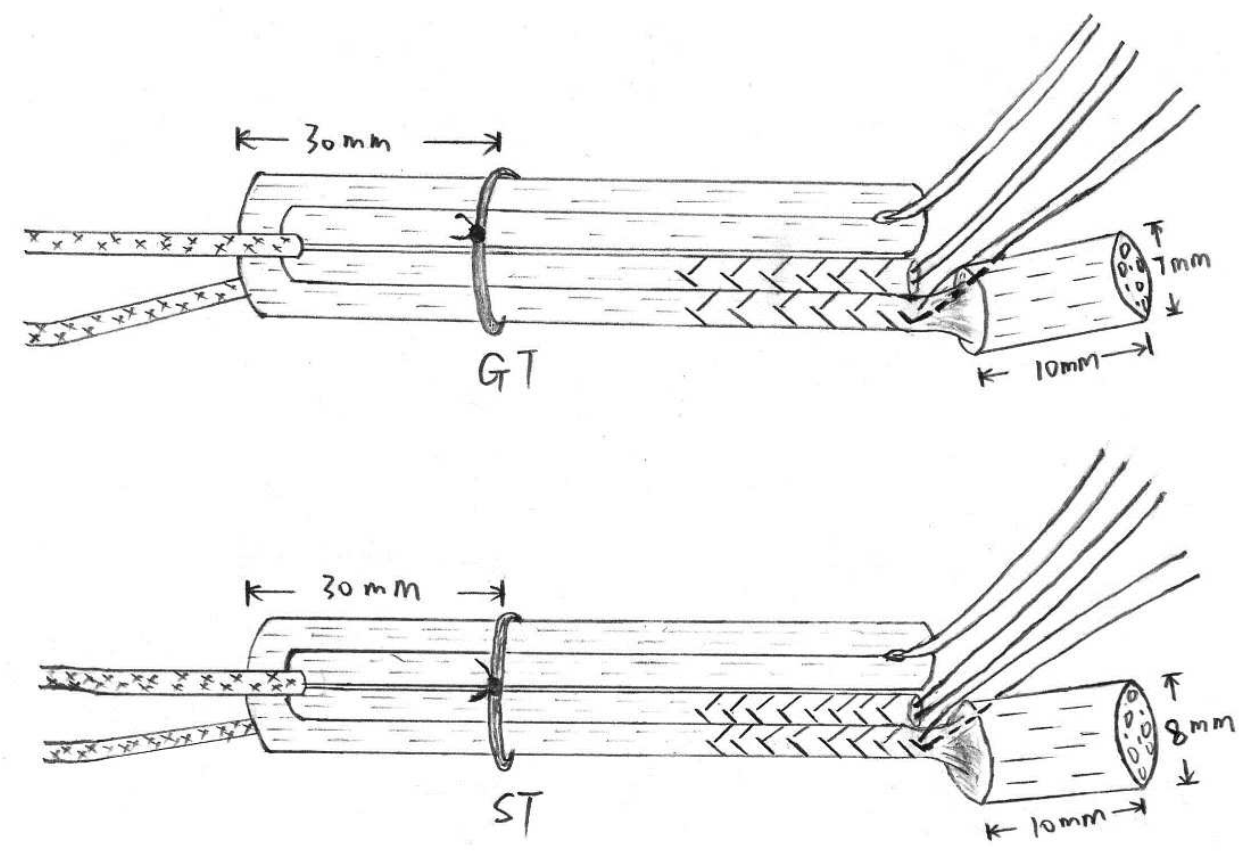

Figure 3B. Initial trim of 2 cube tendon-bone blocks into cylindrical plugs. GT tendon-plug is $10 \mathrm{~mm}$ length and the diameter is $7 \mathrm{~mm}$, and ST tendon-plug is $10 \mathrm{~mm}$ length and the diameter is $8 \mathrm{~mm}$ (the final diameters of the cylindrical plugs are $1 \sim 2 \mathrm{~mm}$ smaller than that of the tibial tunnels). 


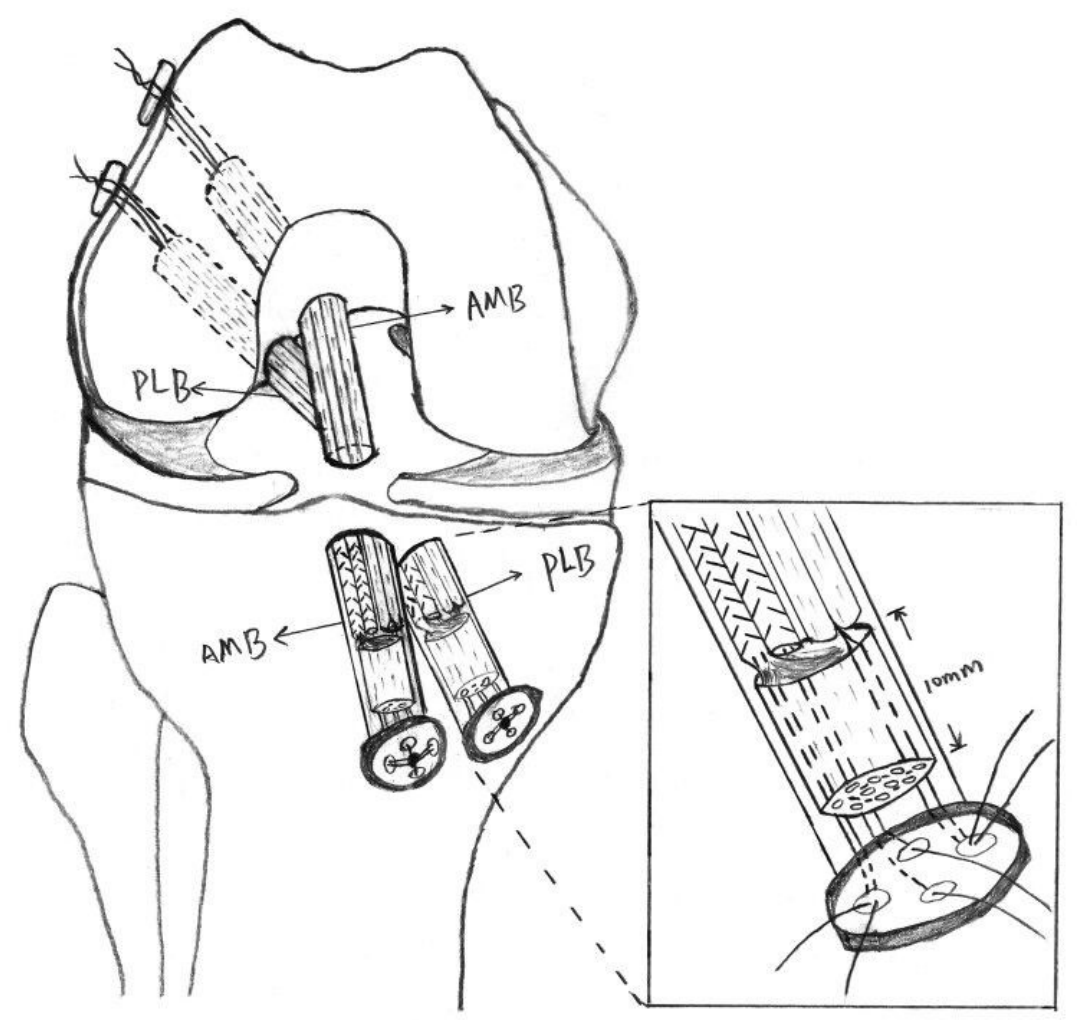

Figure 4. Fixation of the grafts at the femoral and tibial with mini-disc suspension.

The diameter of cylindrical bone plugs were $1 \sim 2 \mathrm{~mm}$ smaller than that of the tibial tunnel, so that the sutures at the end of the graft tendon could be pulled from the tibial tunnel frictionless. AMB: anteromedial bundle, PLB: posterolateral bundle.

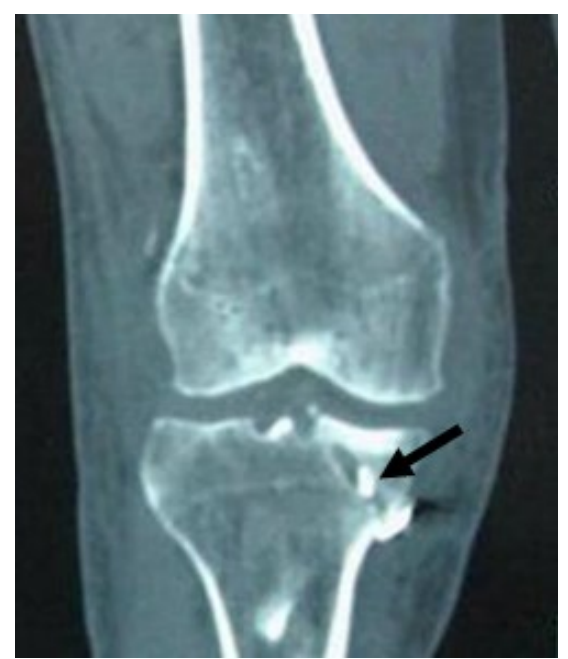

Figure 5. CT (Postoperative, bone plug in the tunnel could be found). 


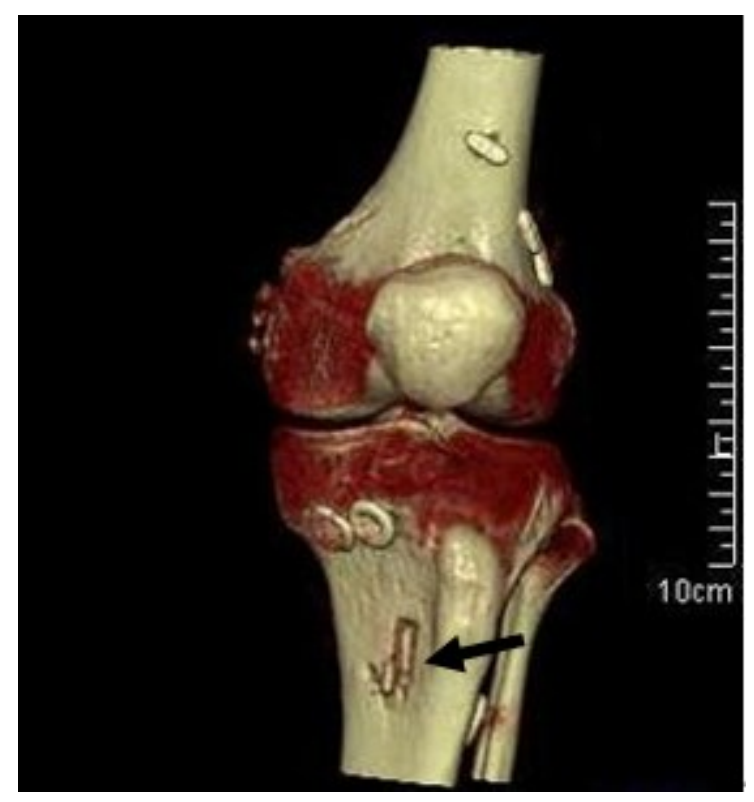

Figure 6A. CT(3 days after operation, bone defect could be found).

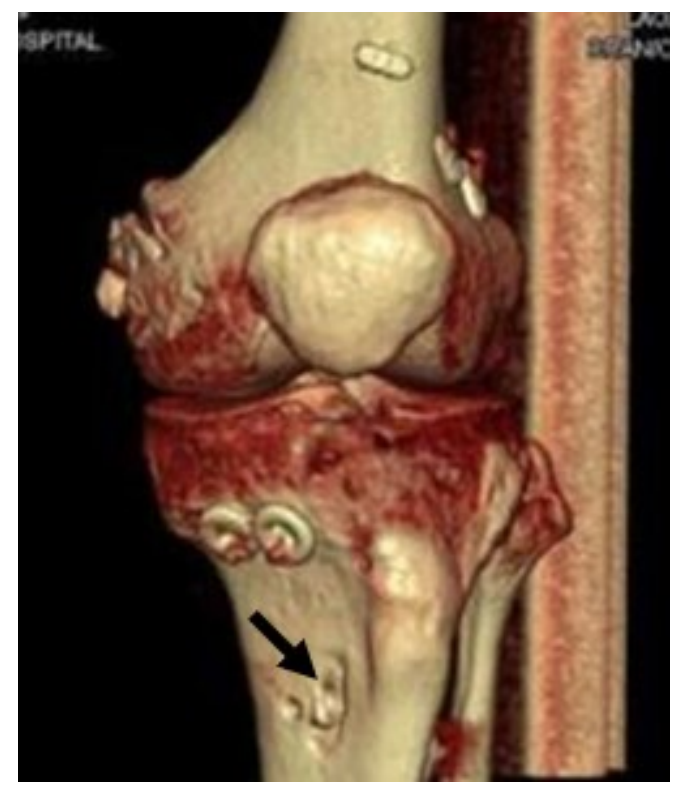

Figure 6B. CT(16 months after operation, bone defect could be healed). 


\section{Reference}

1. Hussin EA, Aldaheri A, Alhabi H, Farouk HA. Modified transtibial versus anteromedial portal techniques for anterior cruciate ligament reconstruction, acomparative study. Open Access J Sports Med. 2018;19:199-213.

2. Adravanti P, Dini F, Girolamo LD, Cattani M, Rosa MA. Single-bundle Versus Doubletunnel Anterior Cruciate Ligament Reconstruction: A Prospective Randomized Controlled Trail with 6-Year Follow-up. J Knee Surg. 2017;30:898-904.

3. Cremer P, Peltier A, Maubisson L, Neyret P, Lustig S, Servien E. Positioning of the Tibial Tunnel After Single-Bundle ACL Primary Reconstruction on 3D CT scans: A New Method. Arthrosc Sports Med Rehabil. 2020;2:e615-22.

4. Zhao JZ. Anatomical single-bundle transtibial Anterior Cruciate Ligament Reconstruction. Arthrosc Tech. 2020;29:e1275-82.

5. Xiang XX, Qu ZN, Sun HL, Ma XJ, Wang WM, Huang LX. Single-tunnel anatomic double-bundle anterior cruciate ligament reconstruction has the same effectiveness as double femoral, double tibial tunnel: A prospective randomized study. Medicine (Baltimore). 2019;98:e14851.

6. Mayr HO, Bruder S, Hube R, Bernstein A, Suedkamp NP, Stoehr A. Single-Bundle Versus Double-Bundle Anterior Cruciate Ligament Reconstruction-5-Year Results. Arthroscopy. 2018;34:2647-53.

7. Aga C, Risberg MA, Fagerland MW, Johansen S, Troan I, Heir S, et al. No Difference in the KOOS Quality of Life Subscore Between Anatomic Double-Bundle and Anatomic Single-Bundle Anterior Cruciate Ligament Reconstruction of the Knee: A Prospective Randomized Controlled Trial With 2 Years' Follow-up. Am J Sports Med. 2018;46:2341-54.

8. Streich NA, Friedrich K, Gotterbarm T, Schmitt H. Reconstruction of the ACL with a semitendinosus tendon graft: a prospective randomized single blinded comparison of double-bundle versus single-bundle technique in male athletes. Knee Surg Sports Traumatol Arthrosc. 2008;16:232-8.

9. Adachi N, Ochi M, Uchio Y, Iwasa J, Kuriwaka M, Ito Y. Reconstruction of the anterior cruciate ligament. Single- versus double-bundle multistranded hamstring tendons. The Journal of Bone and Joint Surgery. 2004;86:515-20.

10. Kassem MS, Motawea BA, Rafalla AA. Anatomic single-bundle versus anatomic double-bundle anterior cruciate ligament reconstruction: a comparative study based on midterm results. The Egyption Orthopaedic Orthopaedic Journal. 2018;53:331-40.

11. Shaver JC, Johnson DL. Revision Anatomic Double-Bundle Anterior Cruciate Ligament Surgery. Operative Techniques in Sports Medicine. 2008;16:157-64.

12. Espejo-Baena A, Serrano-Fernandez JM, Torre-Solis F, Irizar-Jimenez S. Anatomic double-bundle ACL reconstruction with femoral cortical bone bridge support using hamstrings. Knee Surg Sports Traumatol Arthrosc, 2009;17:157-61.

13. Mae T, Shino K, Matsumoto N, Hamada M, Yoneda M, Nakata K. Anatomical twobundle versus Rosenberg's isometric bi-socket $A C L$ reconstruction: a biomechanical comparison in laxity match pretension. Knee Surg Sports Traumatol Arthrosc. 2007; 15:328-34. 
14. Yonetani Y, Toritsuka Y, Yamada Y, Iwahashi T, Yoshikawa H, Shino K. Graft Length Changes in the Bi-Socket Anterior Cruciate Ligament Reconstruction: Comparison Between Isometric and Anatomic Femoral Tunnel Placement. Arthroscopy: The Journal of Arthroscopy. 2005;21:1317-22.

15. Lim CR, Henson T, Ebert J, Annear P. Anterior cruciate ligament reconstruction using a double bundle hamstring autograft configuration in patients under 30 years. World J Orthop. 2019;10:446-53.

16. Mohtadi N, Chan D, Barber R, Paolucci EO. Reruptures, Reinjuries, and Revisions at a Minimum 2-Year Follow-up: A Randomized Clinical Trial Comparing 3 Graft Types for ACL Reconstruction. Clin J Sport Med. 2016;26:96-107.

17. Tiamklang T, Sumanont S, Foocharoen T, Laopaiboon M. Double-bundle versus single-bundle reconstruction for anterior cruciate ligament rupture in adults. Cochrane Database Syst Rev. 2012;11:CD008413.

18. Kondo E, Yasuda K, Azuma H, Tanabe Y, Yagi T. Prospective clinical comparisons of anatomic double-bundle versus single-bundle anterior cruciate ligament reconstruction procedures in 328 consecutive patients. Am J Sports Med. 2008;36: 1675-87.

19. Magnussen RA, Trojani C, Granan LP, Neyret P, Colombet P, Engebretsen L, et al. Patient demographics and surgical characteristics in ACL revision: a comparison of French, Norwegian, and North American cohorts. Knee Surg Sports Traumatol Arthrosc. 2015;23:2339-48.

20. Papageorgiou CD, Ma CB, Abramowitch SD, Clineff TD, Woo SL. A multidisciplinary study of the healing of an intraarticular anterior cruciate ligament graft in a goat model. Am J Sports Med. 2001;29:620-6.

21. Weiler A, Hoffmann RF, Bail HJ, Rehm O, Sudkamp NP. Tendon healing in a bone tunnel. Part II: Histologic analysis after biodegradable interference fit fixation in a model of anterior cruciate ligament reconstruction in sheep. Arthroscopy. 2002;18: 124-35.

22. Irrgang JJ, Anderson AF, Boland AL, Harner CD, Kurosaka M, Neyret $\mathrm{P}$, et al. Development and validation of the international knee documentation committee subjective knee form. Am J Sports Med. 2001;29:600-13.

23. Williams GN, Taylor DC, Gangel TJ, Uhorchak JM, Arciero RA. Comparison of the single assessment numeric evaluation method and the Lysholm score. Clin Orthop Relat Res. 2000;373:184-92.

24. Briggs KK, Lysholm J, Tegner Y, Rodkey W, Kocher MS, Steadman JR. The reliability, validity, and responsiveness of the Lysholm score and Tegner activity scale for anterior cruciate ligament injuries of the knee: 25 years later. Am J Sports Med. 2009;37:8907.

25. Jarvela T, Moisala AS, Paakkala T, Paakkala A. Tunnel enlargement after doublebundle anterior cruciate ligament reconstruction: a prospective, randomized study. Arthroscopy. 2008;24:1349-57.

26. Aga C, Wilson KJ, Johansen S, Dornan G, Prada RF, Engebretsen L. Tunnel widening in single- versus double-bundle anterior cruciate ligament reconstructed knees. Knee Surg Sports Traumatol Arthrosc. 2017;25:1316-27. 
27. Sasaki S, Tsuda E, Hiraga Y, Yamamoto Y, Maeda S, Sasaki E, et al. Prospective Randomized Study of Objective and Subjective Clinical Results Between DoubleBundle and Single-Bundle Anterior Cruciate Ligament Reconstruction. Am J Sports Med. 2016;44:855-64.

28. Robbe R, Paletta GA. Soft-tissue graft fixation in anterior cruciate ligament reconstruction. Operative Techniques in Sports Medicine. 2004;12:188-94.

29. Lu HB, Chen C, Xie SS, Tang TF, Qu J. Tendon Healing in Bone Tunnel after Human Anterior Cruciate Ligament Reconstruction: A Systematic Review of Histological Results. J Knee Surg. 2019;32:454-62.

30. Lazarides AL, Eward WC, Green K, Cardona DM, Brigman B, Taylor DC. Histological Evaluation of Tendon-Bone Healing of an Anterior Cruciate Ligament Hamstring Graft in a 14-Year-Old Boy. Am J Sports Med. 2015;43:1935-40.

31. Tian SQ, Wang YH, Wang B, Liu I, Ha CZ, Li QC, et al. Anatomic Double-Bundle Anterior Cruciate Ligament Reconstruction With a Hamstring Tendon Autograft and Fresh-Frozen Allograft: A Prospective, Randomized, and Controlled Study. Arthroscopy. 2016;32:2521-31.

32. Rodeo SA, Kawamura S, Kim HJ, Dynybil C, Ying L. Tendon healing in a bone tunnel differs at the tunnel entrance versus the tunnel exit: an effect of graft-tunnel motion? Am J Sports Med. 2006;34:1790-800.

33. Tsuda E, Fukuda Y, Loh JC, Debski RE, Fu FH, Woo SL. The effect of soft-tissue graft fixation in anterior cruciate ligament reconstruction on graft-tunnel motion under anterior tibial loading. Arthroscopy. 2002;18:960-7.

34. Shino K, Kawasaki T, Hirose H, Gotoh I, Inoue M, Ono K. Replacement of the anterior cruciate ligament by an allogeneic tendon graft. An experimental study in the dog. J Bone Joint Surg Br. 1984;66:672-81.

35. Webster KE, Feller JA, Hameister KA. Bone tunnel enlargement following anterior cruciate ligament reconstruction: a randomised comparison of hamstring and patellar tendon grafts with 2-year follow-up. Knee Surg Sports Traumatol Arthrosc. 2001; 9: 86-91. 


\section{Figures}

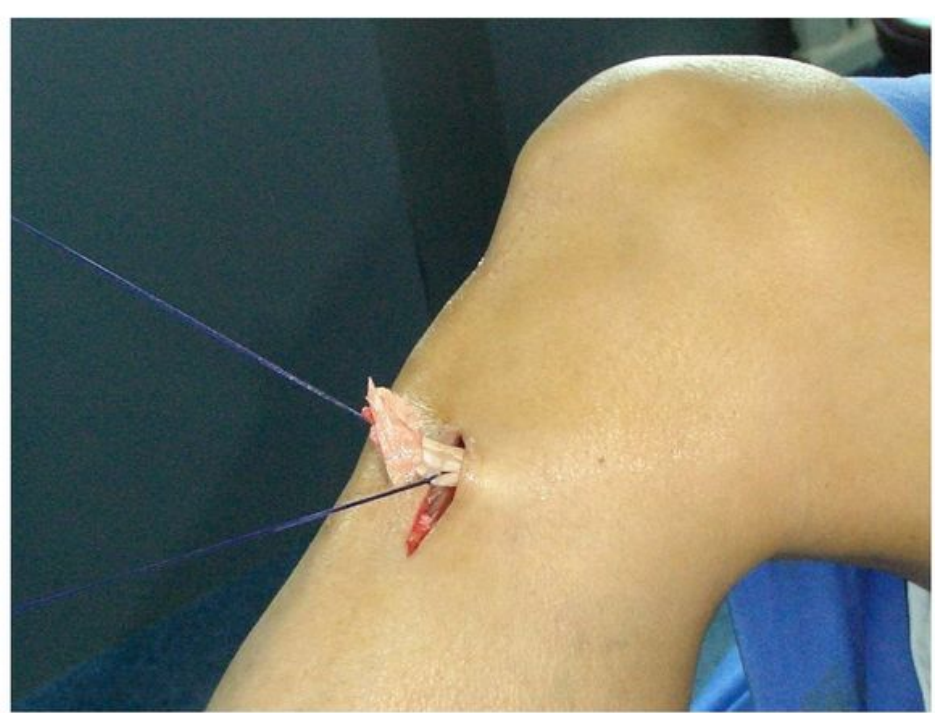

$1 \mathrm{~A}$

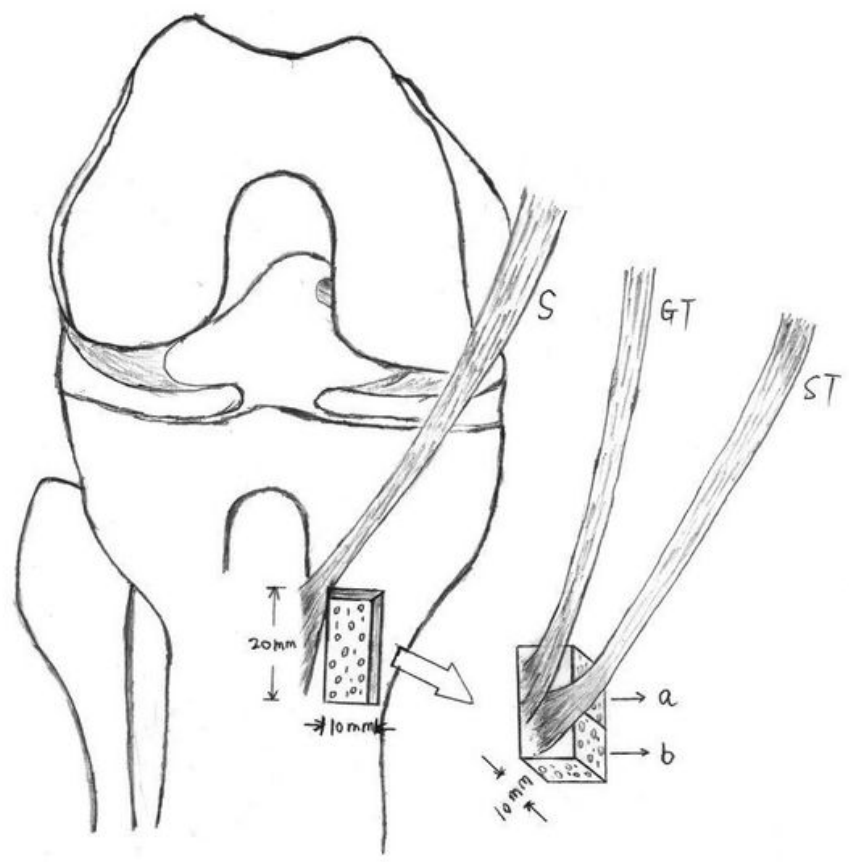

1B

\section{Figure 1}

1A. Harvest of cuboid bone block at the footprint of GT and ST. GT: gracilis tendon; ST: semitendinosus tendon. 1B. The block is $20 \mathrm{~mm}$ length, $10 \mathrm{~mm}$ width, and $10 \mathrm{~mm}$ thickness, and it is cut into 2 equal pieces $(a, b)$.

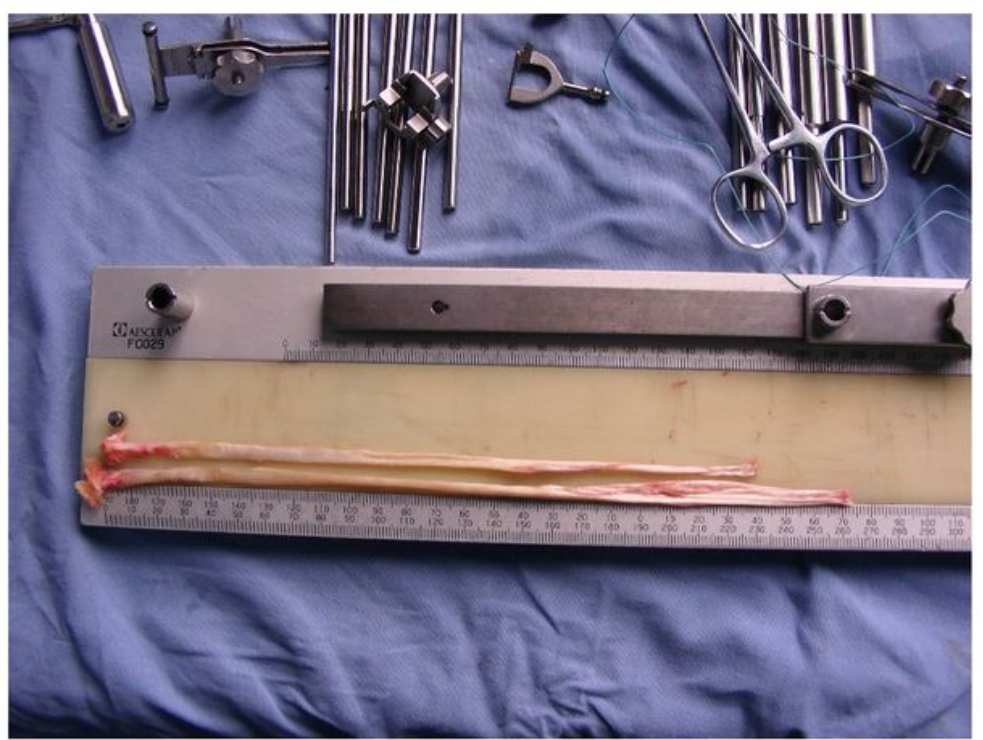

2A
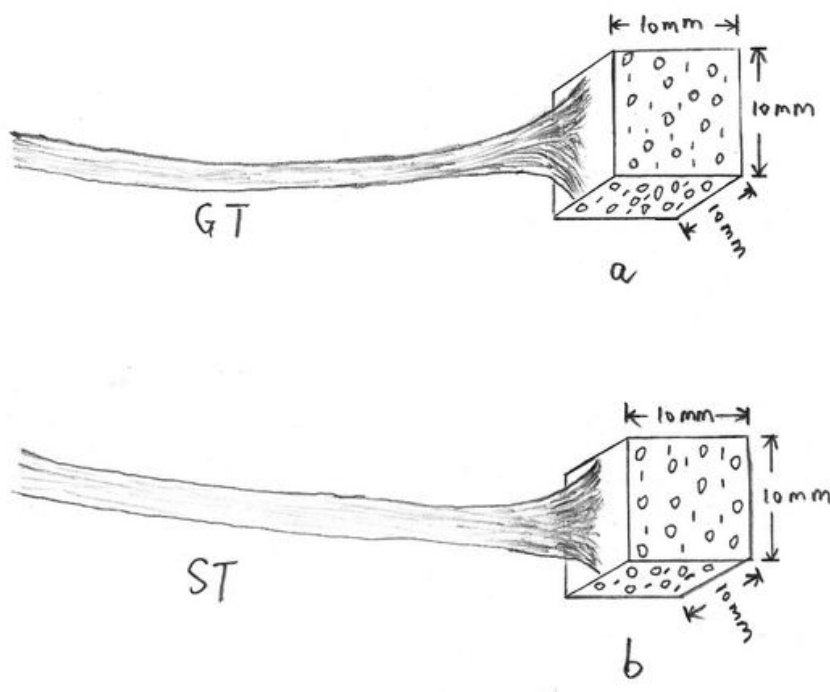

2B

Figure 2 
2A. Harvest of ST and GT, and the bone block is divided into two equal parts. 2B. The bone block is divided into two equal parts. The blocks are $10 \mathrm{~mm}$ length, $10 \mathrm{~mm}$ width, and $10 \mathrm{~mm}$ thickness, respectively.

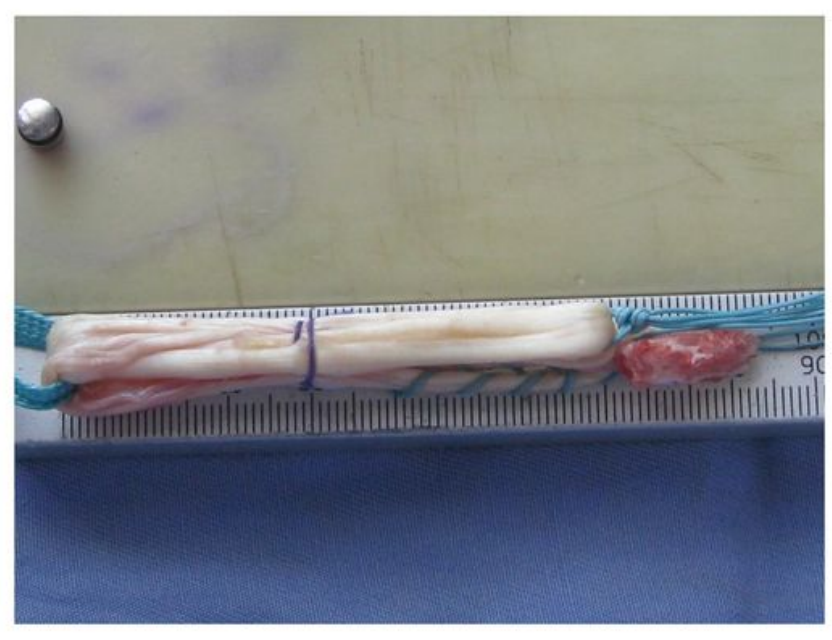

$3 \mathbf{A}$
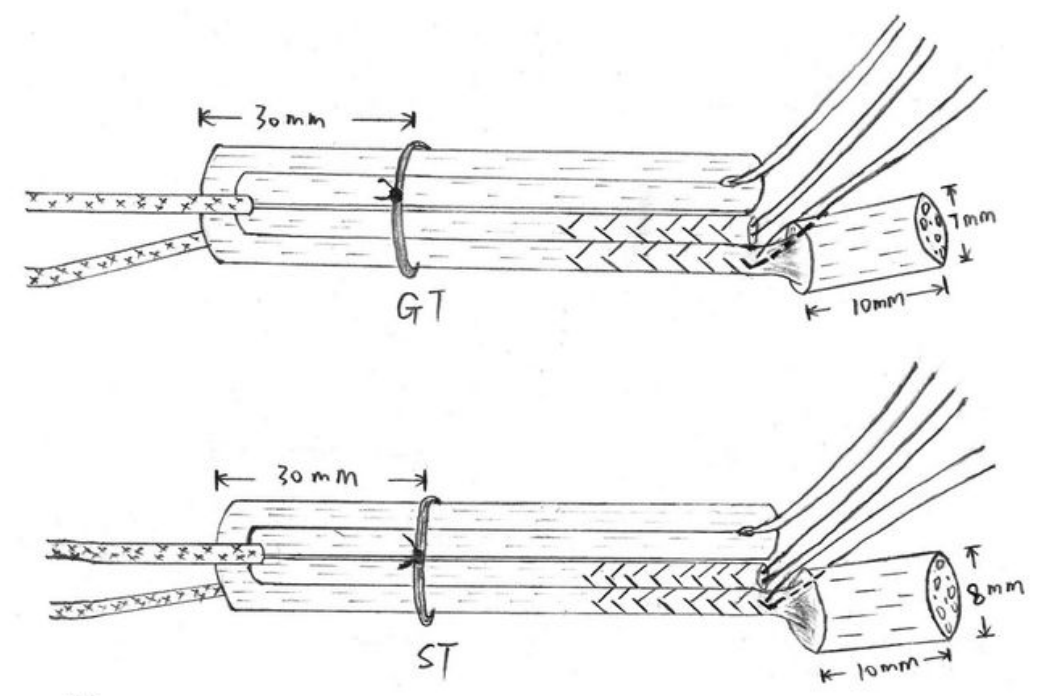

3B

\section{Figure 3}

3A. Suture and fold of GT and ST. 3B. Initial trim of 2 cube tendon-bone blocks into cylindrical plugs. GT tendon-plug is $10 \mathrm{~mm}$ length and the diameter is $7 \mathrm{~mm}$, and ST tendon-plug is $10 \mathrm{~mm}$ length and the diameter is $8 \mathrm{~mm}$ (the final diameters of the cylindrical plugs are $1 \otimes 2 \mathrm{~mm}$ smaller than that of the tibial tunnels). 


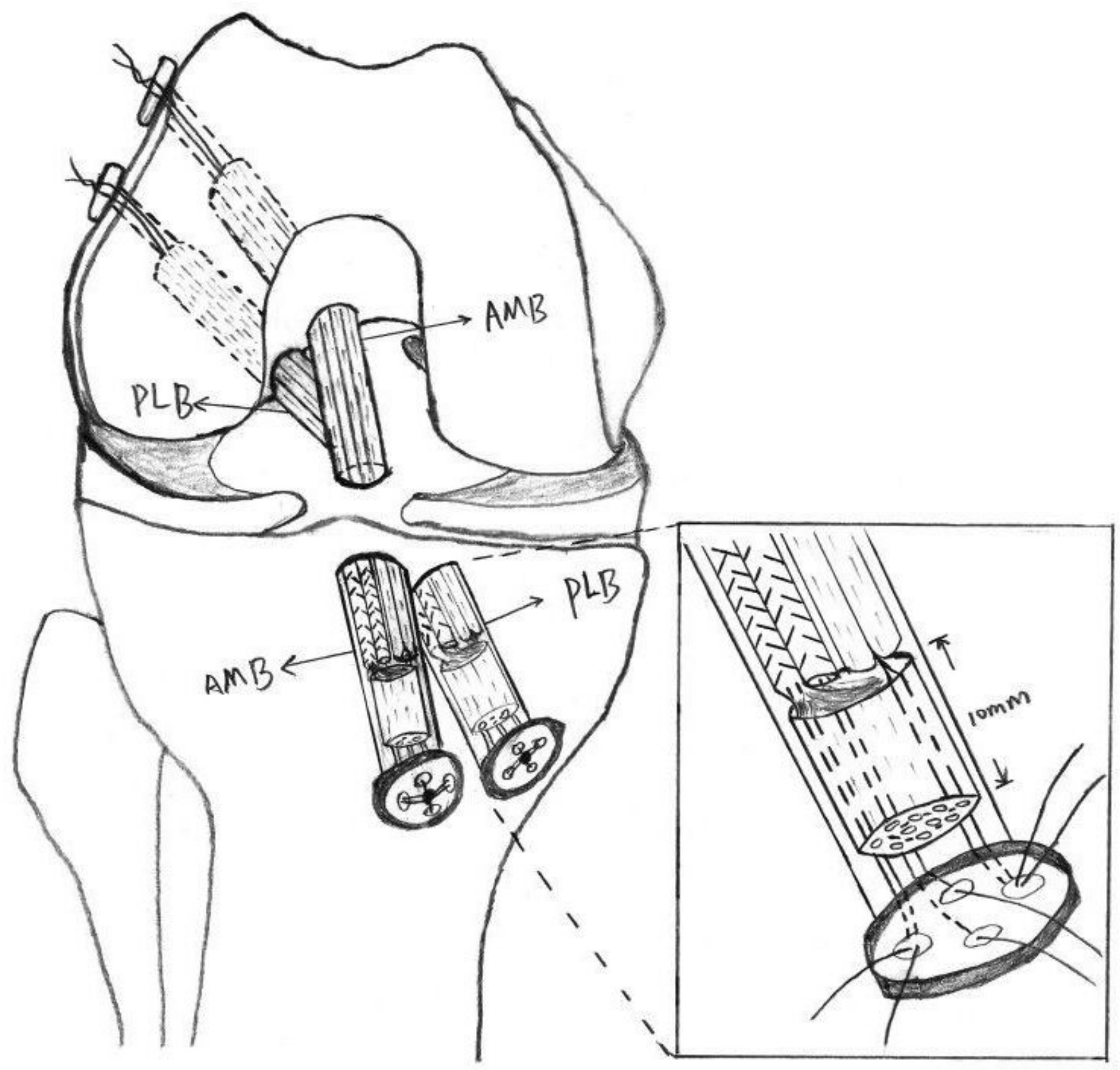

Figure 4

Fixation of the grafts at the femoral and tibial with mini-disc suspension. The diameter of cylindrical bone plugs were $1 \otimes 2 \mathrm{~mm}$ smaller than that of the tibial tunnel, so that the sutures at the end of the graft tendon could be pulled from the tibial tunnel frictionless. AMB: anteromedial bundle, PLB: posterolateral bundle. 


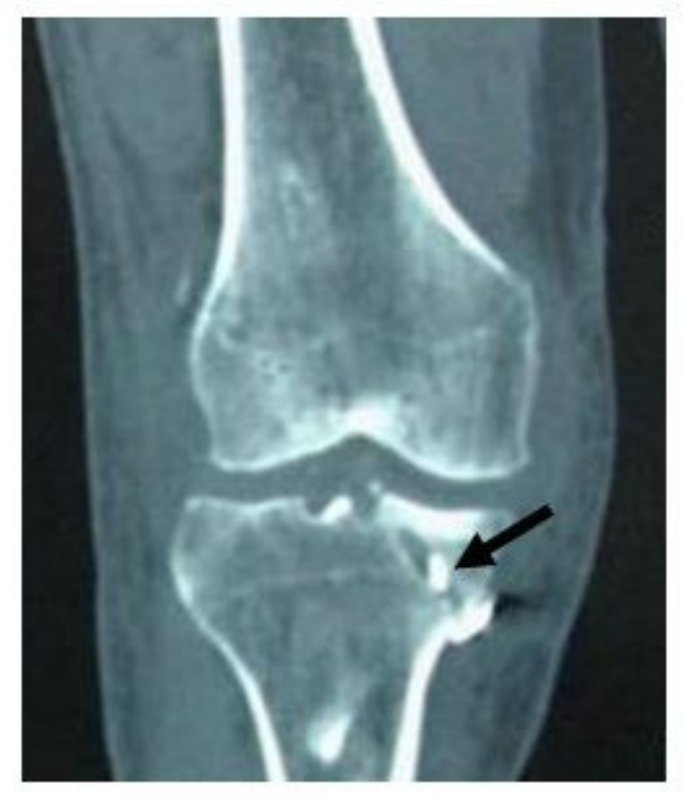

Figure 5

CT (Postoperative, bone plug in the tunnel could be found).
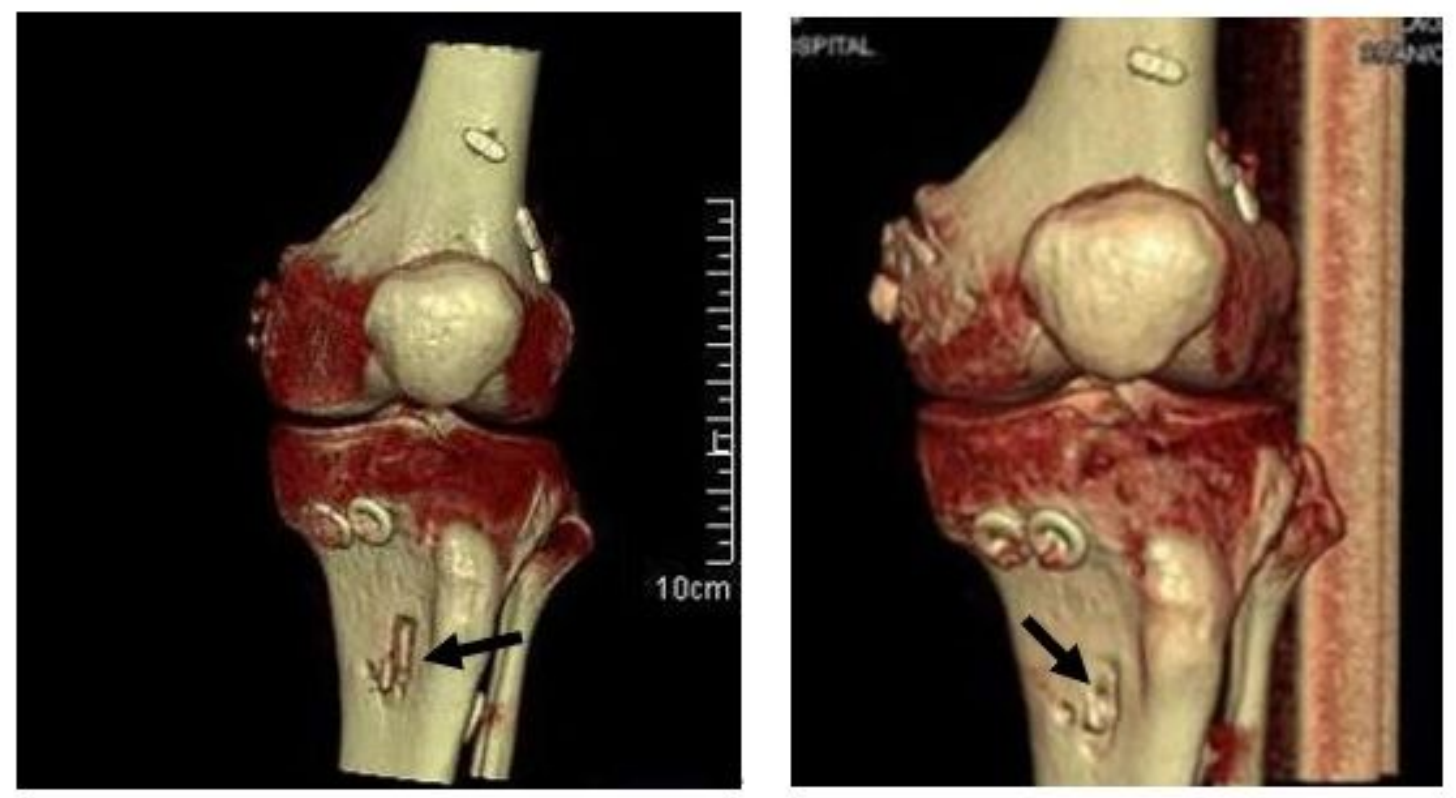

$6 \mathrm{~A}$

6B

Figure 6

6A. СT(3 days after operation, bone defect could be found). 6B. CT(16 months after operation, bone defect could be healed). 